\title{
Submitted: High-resolution ultrasound of the ankles in Lofgren Accepted: syndrome: attention to detail may be the key to diagnosis
} 03.06.2020

Published: 28.09.2020

\author{
Keywords \\ ankle tenosynovitis, \\ edema, \\ arthritis, \\ Lofgren syndrome, \\ acute sarcoidosis
}

\author{
Eleni E. Drakonaki ${ }^{1}$, Emmanouil K. Symvoulakis²$^{2}$,John Gliatis ${ }^{3}$ \\ ${ }^{1}$ Consultant Radiologist, European University of Cyprus Medical School, Engomi, Nicosia, \\ Cyprus \\ ${ }^{2}$ Clinic of Social and Family Medicine, Faculty of Medicine, University of Crete, Greece \\ ${ }^{3}$ University of Patras School of Health Sciences, Department of Medicine, Panepistimioupolis, \\ Greece \\ Correspondence: Drakonaki Eleni MD, PhD, Medical School of the European University \\ of Cyprus, Engomi, Nicosia,Cyprus; tel.00306932399087,e-mail: drakonaki@yahoo.gr
}

DOI: $10.15557 / \mathrm{JoU} .2020 .0035$

\begin{abstract}
We report the case of a 40-year-old man presenting at the emergency department with ankle and feet edema due to acute sarcoidosis (Lofgren syndrome). The diagnosis was suggested based on an ultrasound examination showing bilateral asymmetrical tenosynovitis of the flexor tendons with hypervascular subcutaneous cellulitis without any significant ankle and foot joint effusion or synovitis. This case report highlights the crucial role of ultrasound imaging as the first-line diagnostic tool in the investigation of a non-specific clinical condition, when performed by a knowledgeable sonographer with attention to detail. Radiologists performing ultrasound should be aware of the presenting ultrasound pattern of acute soft tissue sarcoidosis, and should include this entity in the differential diagnosis of patients with distal lower extremity swelling, tenosynovitis and hypervascular subcutaneous cellulitis, in order to narrow down the differential diagnosis and facilitate the clinical workup of the patient.
\end{abstract}

\section{Case report}

A 40-year-old man presented with recent painless asymmetric soft tissue swelling at the ankles and feet predominantly affecting the medial side of the left foot (Fig. 1). The clinical examination was otherwise unremarkable, and there was no fever, signs of infection or heart failure.

He was referred to the radiology department for an ultrasound (US) examination of the ankles and foot, which revealed bilateral tenosynovitis of the tibialis posterior and flexor digitorum longus tendons (Fig. 2) with subcutaneous small fluid collections, in keeping with fat cellulitis, and increased vascularity of subcutaneous fat consistent with acute inflammation (Fig. 3). The findings were bilateral, but asymmetrical. There was no effusion or synovitis in the ankle joint and the midtarsal joints (Fig. 4). The remaining ankle tendons were intact. There were no signs of deep venous thrombosis or superficial vein insufficiency. At this stage, the suspicion of Lofgren syndrome was raised by the radiologist based on the coexistence of subcutaneous edema and tenosynovitis without evidence of arthritis.

A chest X-ray and blood tests were subsequently performed. The chest X-ray revealed right paratracheal and bilateral symmetrical hilar enlargement, but no lung field abnormality was evident (Fig. 5). Blood tests showed normal RBC, WBC and platelets counts, and mildly increased erythrocyte sedimentation rate (ESR $48 \mathrm{~mm} 1^{\text {st }}$ hour) and C-reactive protein (CRP $35 \mathrm{mg} / \mathrm{l}$, normal value: $<5 \mathrm{mg} / \mathrm{l}$ ). A CT scan of the lung confirmed the presence of hilar lymph node enlargement, and revealed a few small nodules with perilymphatic distribution (Fig. 6).

Bronchoscopy was performed, and bronchoalveolar lavage confirmed the presence of non-caseating granulomas and CD4+ lymphocytic alveolitis, which was consistent with pulmonary sarcoidosis. Based on the above findings, the 


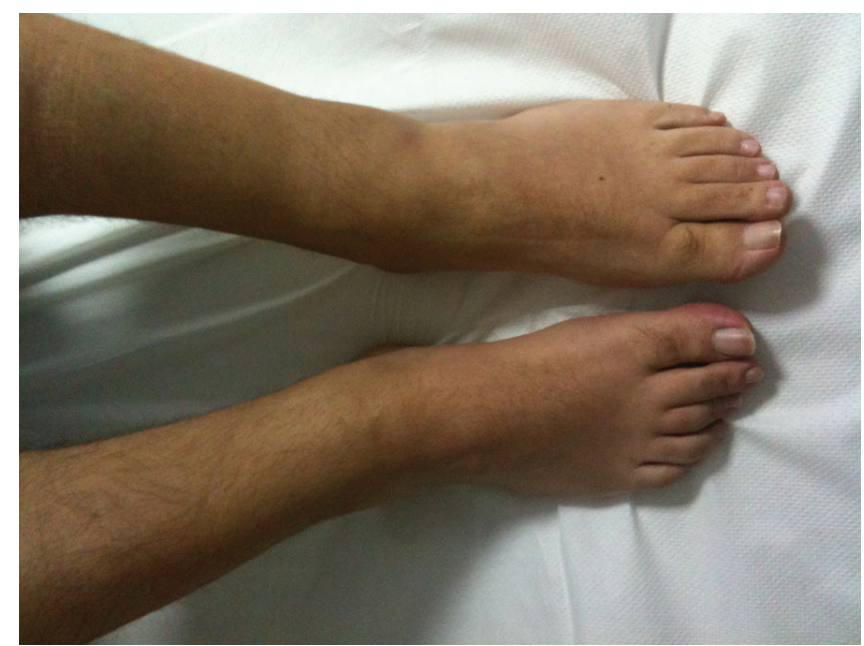

Fig. 1. Clinical photograph at presentation showing soft tissue edema of the left ankle and foot

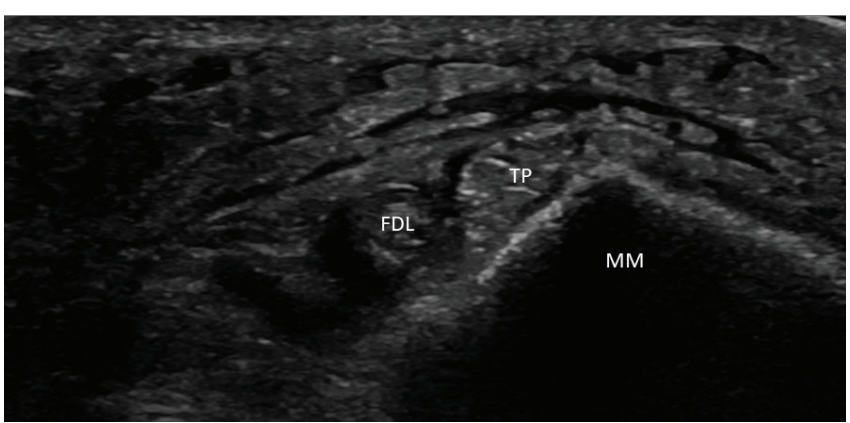

Fig. 2. Axial ultrasound image of the flexor tendons at the level of the left medial malleolus (MM), showing hypoechoic fluid around the tibialis posterior tendon (TP) and the flexor digitorum longus (FDL) tendon, in keeping with tenosynovitis. There are also extensive subcutaneous small fluid collections consistent with subcutaneous edema/cellulitis

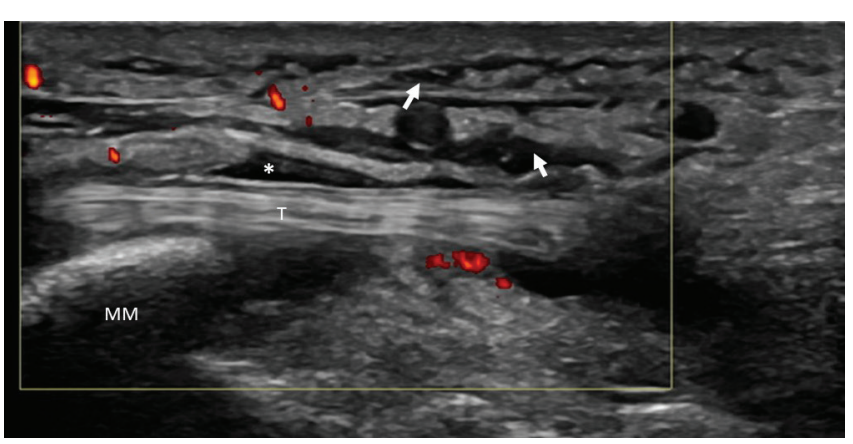

Fig. 3. Longitudinal ultrasound image with color Doppler of the tibialis posterior tendon (T) at the level of the left medial malleolus (MM). There is effusion (asterisk) in the tendon sheath, consistent with tenosynovitis. There are also subcutaneous small fluid collections (arrow), in keeping with fat cellulitis, and increased vascularity of the subcutaneous fat consistent with acute inflammation

diagnosis of acute sarcoidosis with soft tissue involvement (Lofgren syndrome) was established. The patient was treated with oral steroids, and the soft tissue edema completely resolved after a few days of treatment.

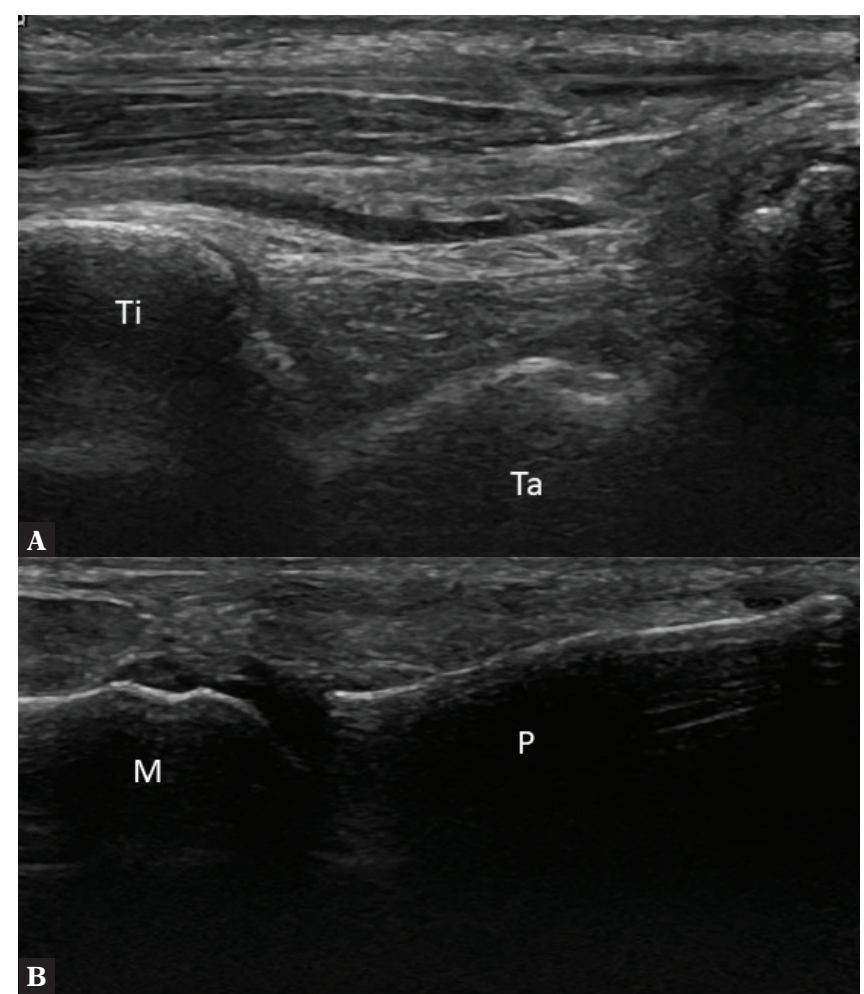

Fig. 4. A. Longitudinal ultrasound image of the anterior recess of the left tibiotalar joint and $\mathbf{B}$. the metatarsophalangeal joint showing that there is no significant effusion or synovitis. Ti-Tibia, Ta-Talus, $M$-metatarsal bone, $P$ - phalangeal bone

The patient has provided his written informed consent for his data and images to be published.

\section{Discussion}

Lofgren syndrome is an acute form of sarcoidosis first described by S. Lofgren in $1952^{(1)}$. Sarcoidosis, an inflammatory disease characterized by an increased CD4 T cell immune response, affects various organs including the musculoskeletal system, where it has a polymorphic appearance, including nonspecific arthralgia, polyarthritis, and Lofgren syndrome ${ }^{(1-4)}$. The originally described typical diagnostic triad included symmetrical ankle arthritis, hilar lymphadenopathy, and erythema nodosum ${ }^{(1)}$. However, erythema nodosum, a painful disorder of subcutaneous fat, may be absent in patients with Lofgren syndrome, as was in our case $^{(2,5,6)}$. Contrary to the typical diagnostic features originally described, in the era of US imaging it has been shown that mild joint effusion is a rare cause of ankle swelling in Lofgren syndrome, found in only $23-25 \%$ of $\operatorname{cases}^{(5,6)}$. It has been originally suggested by a small number of papers that tenosynovitis of the peroneal, flexor and extensor tendons is the main feature responsible for ankle and foot swelling and periarthritis $^{(2-5)}$. Specifically, hypervascular subcutaneous edema and tenosynovitis are found in $83-92 \%$ and $33-39 \%$ of Lofgren syndrome cases, respectively ${ }^{(5,6)}$. In line with the findings of the early US studies ${ }^{(2-5)}$, periarthritis with signifiant subcutaneous edema and tenosynovitis has been observed as the predominant feature of Lofgren syndrome 


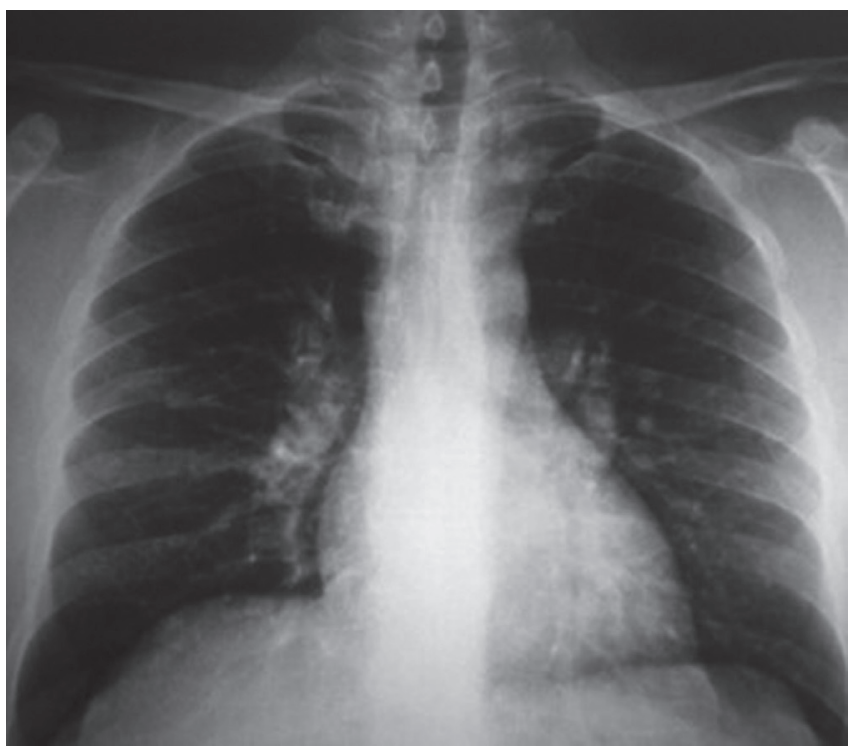

Fig. 5. Posteroanterior chest X-ray showing bilateral lobular hilum enlargement, suggesting hilar lymphadenopathy

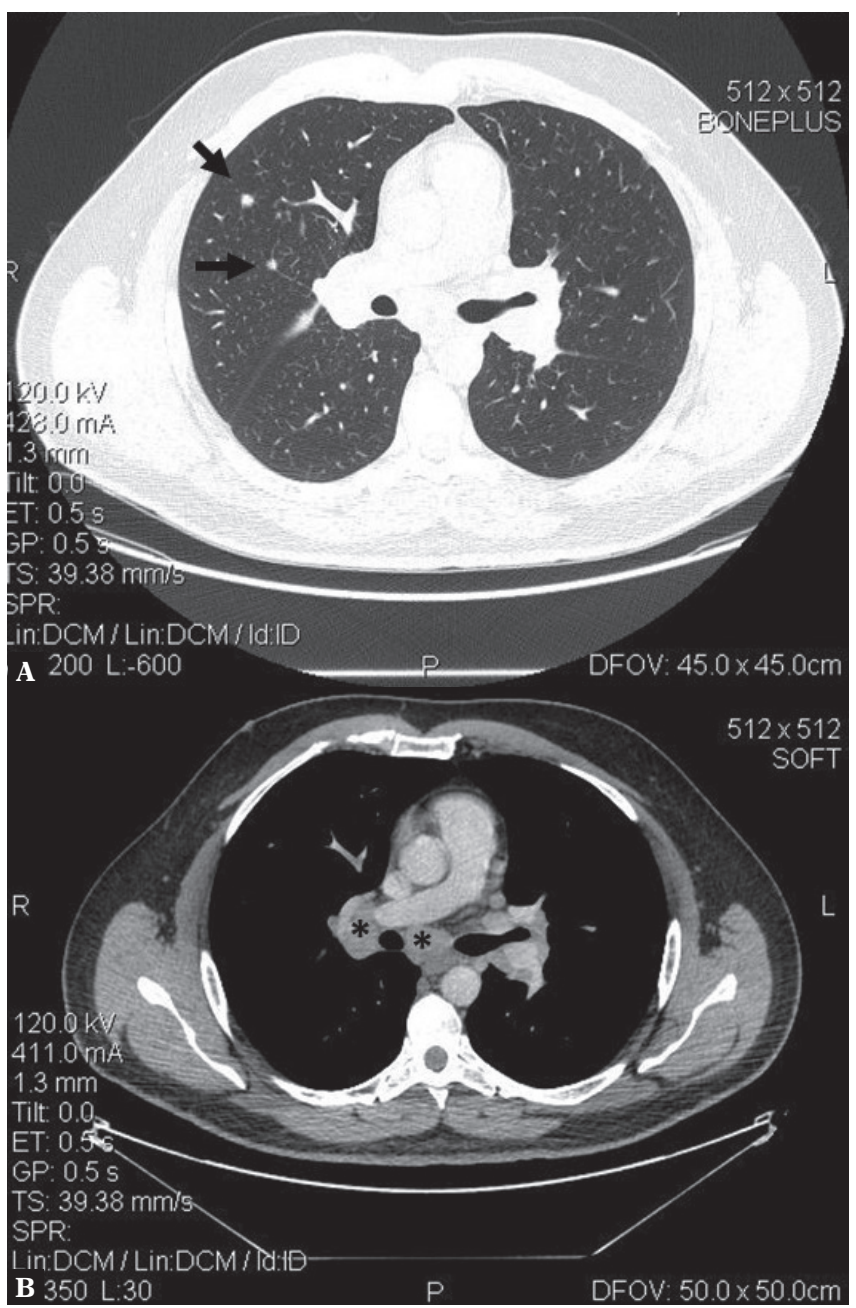

Fig. 6. Axial CT images of the chest in lung (A) and mediastinal (B) windows, showing the presence of enlarged paratracheal and mediastinal nodes (asterisks) and parenchymal nodules with perilymphatic distribution (arrows) in two more recent cohort studies of 36 and 40 cases examined using high-resolution US with Doppler ${ }^{(6,7)}$. They both confirmed that extensive subcutaneous edema is the major reason for ankle swelling in Lofgren syndrome, and mild tenosynovitis is a very common associated finding, whereas distinct articular synovitis suggesting arthritis is rare, and if it is present, then only to a mild degree, without relevant power Doppler activity ${ }^{(6,7)}$. It is therefore suggested that the inclusion of bilateral ankle arthritis among the diagnostic criteria for Lofgren syndrome deserves reappraisal ${ }^{(7)}$. Thus, the coexistence of tenosynovitis with cellulitis may be the first manifestation and the dominant presenting feature of acute sarcoidosis in patients who have no prior history of sarcoidosis or inflammatory arthritis ${ }^{(2,4-7)}$.

Subcutaneous edema with vascularity and tendinosis may also be present in other conditions, and not just sarcoid. Subcutaneous edema may be due to a variety of disorders including hepatic disease, renal deficiency, cardiac failure, and allergic reactions ${ }^{(8)}$. Tendinosis and tenosynovitis may be non-specific features of degenerative, traumatic, biomechanical and rheumatologic disorders, such as inflammatory arthritis. However, the typical combination of subcutaneous edema with hypervascularity and tenosynovitis without sonographic features of arthritis (effusion, synovial proliferation with or without intraarticular hypervascularity) eliminates the possibility of other diagnoses, and facilitates the establishment of the diagnosis of Lofgren syndrome ${ }^{(6,7)}$. Conversely, in patients with ankle swelling, the presence of US findings consistent with distinct arthritis, as stated above, challenges the diagnosis of Lofgren syndrome, and suggests arthritis instead. The diagnosis in our case would have been much more straightforward, if erythema nodosum was present, as it would have appeared as tender, erythematous, subcutaneous nodules typically located symmetrically on the anterior surfaces of the lower extremities ${ }^{(8)}$. In the absence of skin changes, the contribution of US to raising the suspicion of Lofgren syndrome is even more pronounced. On the other hand, the absence of significant ankle and foot joint effusion or synovitis may be an important additional feature to minimize the possibility of inflammatory arthritis in the differential diagnostic workup. Moreover, the additional presence of tenosynovitis limits the differential diagnosis of isolated subcutaneous ankle and foot edema, excluding hepatic, renal, cardiac and allergic conditions ${ }^{(9)}$.

In this case of acute soft tissue sarcoidosis (Lofgren syndrome), the diagnosis was suggested by the US examination of the ankles and foot performed in the emergency setting, thus allowing a shortcut in the workup of the patient to an accurate diagnosis and treatment. The patient presented with a nonspecific appearance of ankle and foot swelling, and US revealed extensive cellulitis and bilateral ankle tenosynovitis without arthritis. Moreover, the absence of significant ankle joint effusion eliminated the possibility of tendon sheath effusion caused by the communication of the tendon sheaths with the ankle joint space, which is a common anatomic variation. The diagnosis of Lofgren syndrome was suggested based on the typical US triad of subcutaneous hypervascular edema with tenosynovitis without evidence of arthritis. The chest X-ray and further testing for sarcoidosis 
were ordered following the US examination to confirm the original suspicion in accordance with the routine work up of all patients under investigation for sarcoidosis.

High-resolution US with Doppler is the imaging test of choice to characterize ankle swelling. This case report highlights the crucial role of US as the first-line imaging method at the emergency setting, provided that it is performed by a highly skilled sonographer who pays attention to details. Soft tissue edema is a common clinical condition which may be caused by a wide spectrum of diseases, thus necessitating an extensive clinical, imaging and laboratory workup in order to shorten the differential diagnosis ${ }^{(9)}$. In this clinical setting, US performed by an experienced musculoskeletal sonographer may allow the depiction of specific US findings and patterns suggesting specific diseases. Therefore, the inclusion of US in the initial workup of patients with ankle and foot soft tissue edema would possibly save time and resources by directing the clinician's attention to a specific spectrum of diseases to be further investigated.

\section{References}

1. Lofgren S, Lundback H: The bilateral hilar lymphoma syndrome; a study of the relation to age and sex in 212 cases. Acta Med Scand 1952; 142: 259-264.

2. Cantini F, Niccoli L, Olivieri I, Barozzi L, Pavlica P, Bozza A et al.: Remitting distal lower extremity swelling with pitting oedema in acute sarcoidosis. Ann Rheum Dis 1997; 56: 565-566.

3. Mañá J, Gómez-Vaquero C, Salazar A, Valverde J, Juanola X, Pujol R: Periarticular ankle sarcoidosis: a variant of Löfgren's syndrome. J Rheumatol 1996; 23: 874-877.

4. Olivieri I, Salvarani C, Cantini F: Remitting distal extremity swelling with pitting edema: a distinct syndrome or a clinical feature of different inflammatory rheumatic diseases? J Rheumatol 1997; 24: 249-252.

5. Kellner H, Späthling S, Herzer P: Ultrasound findings in Löfgren's syndrome: is ankle swelling caused by arthritis, tenosynovitis or periarthritis? J Rheumatol 1992; 19: 38-41.

\section{Conclusion}

In conclusion, this case shows the high diagnostic potential of musculoskeletal sonography. Radiologists performing emergency US examinations should be aware of the presenting features of acute sarcoidosis, and should include this entity in the differential diagnosis of patients with distal lower extremity swelling and US findings of cellulitis and tenosynovitis with or without ankle joint effusion, in order to facilitate the clinical workup of the patient.

\section{Conflict of interest}

Authors do not report any financial or personal connections with other persons or organizations, which might negatively affect the contents of this publication and/or claim authorship rights to this publication.

6. Le Bras E, Ehrenstein B, Fleck M, Hartung W: Evaluation of ankle swelling due to Lofgren's syndrome: a pilot study using B-mode and power Doppler ultrasonography. Arthritis Care Res (Hoboken) 2014; 66: $318-322$

7. Goussault C, Albert JD, Coiffier G, Lamer F, Guillin R, Le Goff B et al.: Ultrasound characterization of ankle involvement in Löfgren syndrome. Joint Bone Spine 2018; 85: 65-69.

8. Trayes KP, Studdiford JS, Pickle S, Tully AS: Edema: diagnosis and management. Am Fam Physician 2013; 88: 102-110.

9. Palmucci S, Torrisi SE, Caltabiano DC, Puglisi S, Lentini V, Grassedonio E et al.: Clinical and radiological features of extra-pulmonary sarcoidosis: a pictorial essay. Insights Imaging 2016; 7: 571-587. 